\title{
Manipulation and Control of Hydrogen Bond Dynamics in Absorbed Ice Nanoclusters
}

\author{
Heiko Gawronski, ${ }^{1}$ Javier Carrasco, ${ }^{2}$ Angelos Michaelides, ${ }^{2,3}$ and Karina Morgenstern ${ }^{1}$ \\ ${ }^{1}$ Institut für Festkörperphysik, Leibniz Universität Hannover, Appelstrasse 2, D-30167 Hannover, Germany \\ ${ }^{2}$ Fritz-Haber-Institut der Max-Planck-Gesellschaft, Faradayweg 4-6, 14195 Berlin, Germany \\ ${ }^{3}$ Materials Simulation Laboratory, London Centre for Nanotechnology and Department of Chemistry, University College London, \\ London WCIE 6BT, United Kingdom \\ (Received 12 June 2007; revised manuscript received 8 November 2007; published 24 September 2008)
}

\begin{abstract}
Inelastic electron tunneling is used to explore the dynamics of ice nanoclusters adsorbed on $\mathrm{Ag}(111)$. The diffusion of entire nanoclusters or internal hydrogen bond rearrangement can be selectively controlled by injecting electrons either directly into the clusters themselves or indirectly ("indirect inelastic electron tunneling") into the substrate at distances of up to $20 \mathrm{~nm}$ from them; a reaction probability that oscillates with the tip-cluster lateral distance presents evidence that surface state electrons mediate the excitation. Density functional theory calculations reveal a strong sensitivity of the computed activation energies of the individual processes to the applied electrical field.
\end{abstract}

The controlled manipulation of atoms and molecules on surfaces not only provides a means to deepen understanding of basic physical and chemical processes at surfaces but also offers technological opportunities through the prospect of building single molecule devices. In this regard, the scanning tunneling microscope (STM) has proved uniquely capable of both imaging and manipulating individual adsorbates. In particular, STM has been used to build nanostructures of molecular assemblies [1] and, through inelastic electron tunneling (IET) [2], effect bond changes of adsorbates on metal surfaces including diffusion, dissociation, and internal bond rearrangement [3-9]. Furthermore, for the example of ammonia on $\mathrm{Cu}(100)$, it was shown how the electronic tunneling current can be tuned to induce either the translation or desorption of individual molecules [7].

In the aforementioned studies, processes were induced by injecting electrons directly into an adsorbate from a STM tip located precisely above it. It is known, however, that "nonlocal" [10] or "indirect" [9] excitation with the STM tip located at some distance from the adsorbates is also possible. Here we report for the case of $\mathrm{D}_{2} \mathrm{O}$ nanoclusters on $\mathrm{Ag}(111)$ that we can selectively control whether entire $\mathrm{D}_{2} \mathrm{O}$ nanoclusters undergo lateral diffusion (translation) or internal hydrogen bond rearrangement by injecting electrons either directly into the clusters or at some lateral distance from them. Indeed, adsorbed clusters can be manipulated at lateral electron injection distances of up to $20 \mathrm{~nm}$. In addition, the probability for cluster rearrangement events oscillates with the tip-cluster lateral distance indicating that surface state electrons mediate the excitation. Density functional theory (DFT) calculations help to rationalize the contrasting behavior of the "direct" and "indirect" IET experiments by revealing a strong dependence of the relative barrier heights of the translation and internal hydrogen bond rearrangement processes on the applied electrical field. Overall, this study demonstrates a novel means for the controlled manipulation of processes at surfaces.

The measurements were performed with a low temperature STM that operates at $5 \mathrm{~K}$ [11]. The $\mathrm{Ag}(111)$ surface was cleaned by cycles of $\mathrm{Ne}^{+}$sputtering and annealing. Ultrapure $\mathrm{D}_{2} \mathrm{O}$ was degassed in vacuum by freeze-pumpthaw cycles. A few percent of a $\mathrm{D}_{2} \mathrm{O}$ bilayer was deposited through a molecular tube doser, and a $23 \mathrm{~s}$ deposition leads to a variety of "amorphous" and "crystalline" clusters of 6-12 molecules $[6,12]$. In particular, the crystalline clusters of 6-9 molecules are stable and easily identifiable with STM on $\mathrm{Ag}(111)$ [and $\mathrm{Cu}(111)$ ] [12]. Their basic building block consists of six molecules arranged in a buckled hexagon. Three of the molecules allow hydrogen-bonded attachment of an additional water leading thus to heptamers, octamers, and nonamers [12]. The STM images of such clusters are dominated by the additional peripheral molecules, each of them imaged as a protrusion. Examples of a heptamer, an octamer, and a nonamer are shown in Figs. 1, 2(a), and 2(b), respectively.

First, we manipulate the $\mathrm{D}_{2} \mathrm{O}$ clusters by exciting them directly with the STM tip. The results for a consecutive series of IET manipulations of a heptamer on $\mathrm{Ag}(111)$ are shown in Fig. 1. Upon ramping the voltage at the positions
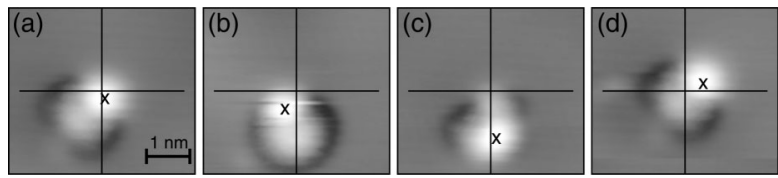

FIG. 1. IET manipulation of a $\mathrm{D}_{2} \mathrm{O}$ heptamer on $\operatorname{Ag}(111)(I=$ $20 \mathrm{pA}, V=-19 \mathrm{mV}, T=5 \mathrm{~K})$. The voltage is ramped from -19 to $-504 \mathrm{mV}$ within $1 \mathrm{~s}$ at the locations indicated by the crosses. Lines serve to guide the eye and are identical in all images. 


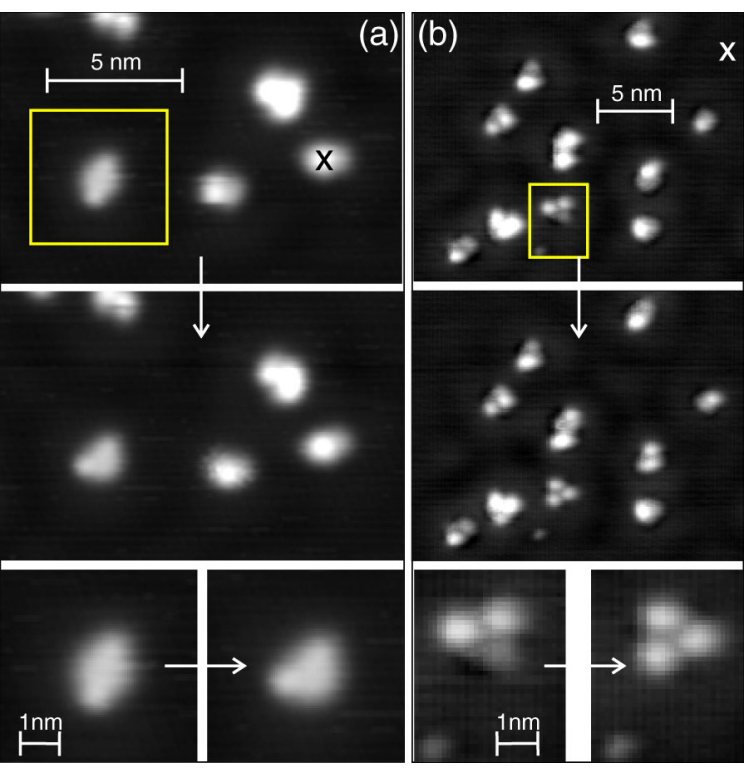

FIG. 2 (color online). Indirect IET manipulations of $\mathrm{D}_{2} \mathrm{O}$ nanoclusters on $\operatorname{Ag}(111)$ showing the surface before (top) and after (middle) manipulation. In (a) the $\mathrm{D}_{2} \mathrm{O}$ octamer indicated by the box transforms during a $0.8 \mathrm{~s} 200 \mathrm{mV}$ pulse at the site marked by the cross $(I=12 \mathrm{pA}, V=103 \mathrm{mV})$. In (b) the $\mathrm{D}_{2} \mathrm{O}$ nonamer indicated by the box transforms during a $3.2 \mathrm{~s} 510 \mathrm{mV}$ pulse at the site marked by the cross $(I=12 \mathrm{pA}, V=72 \mathrm{mV})$. The bottom panels in (a) and (b) show close-up images of the octamer and nonamer before (left) and after (right) manipulation.

indicated, changes are clearly observed in the images of the heptamer. Specifically, the peripheral molecule (bright protrusion) appears to jump between different sites on the underlying hexamer. Also, the entire heptamer diffuses across the surface during the excitation. Thus, the direct IET manipulation effects a number of changes to the adsorbed cluster, which include translation of the entire cluster across the surface and/or rotation along with internal rearrangement of the hydrogen bonds. Also, although not shown in Fig. 1, in the direct IET manipulation experiments the clusters are sometimes transformed to structures which look more amorphous in nature.

Interestingly, we find that it is possible to manipulate the clusters and induce bond rearrangement by injecting electrons not directly into the nanoclusters but at some distance from them. This process, which we call indirect IET [9], is possible by injecting electrons into another cluster as well as into the bare surface as exemplified in Fig. 2(a) for a $\mathrm{D}_{2} \mathrm{O}$ octamer and Fig. 2(b) for a $\mathrm{D}_{2} \mathrm{O}$ nonamer. For the octamer, the two additional molecules (two additional protrusions) are initially in the para orientation; i.e., they are bound to opposite water molecules in the underlying hexamer. Indirect IET leads to a change into the meta orientation, i.e., adsorption of the additional molecules to next-nearest-neighboring water molecules of the hexamer. A collective rearrangement of the additional molecules of a nonamer is shown in Fig. 2(b). For indirect IET, in contrast to direct IET, the crystalline clusters are never caused to translate across the surface nor are they changed to amorphous clusters, which sometimes happens with direct IET.

Since the main difference between direct and indirect IET is that the field at the excited molecule is reduced considerably in indirect IET [13], we now use DFT to explore the sensitivity of the water cluster dynamics to electric field effects [14]. Having recently established the structures of the adsorbed clusters on this surface [12], the focus here was on possible cluster rearrangement and translation processes. Specifically, translation of an entire heptamer across the surface and transfer of the peripheral water between adjacent sites of the heptamer were considered, since the experiments implicate the former in direct IET manipulation and the latter in indirect IET. The results of these calculations are summarized in Fig. 3, which shows the variation in the computed activation energies of the two processes as a function of applied electrical field. At zero applied field, the barrier for translation of the heptamer as a whole is at $0.19 \mathrm{eV}$ slightly larger than the barrier of $0.16 \mathrm{eV}$ for the peripheral water to hop between adjacent sites of the heptamer. This gives an indication that, in the absence of an applied field, internal reorientation of the cluster by means of a hop of the peripheral molecule from one site to another is more facile than translation of the entire water cluster across the surface. Of course, the energy difference between the two com-

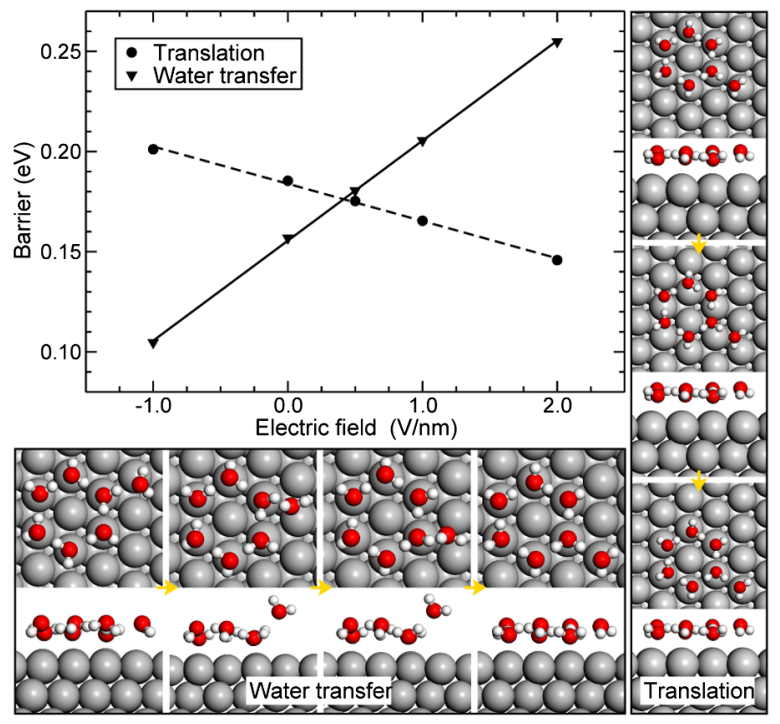

FIG. 3 (color online). Dependence of the computed DFT reaction barriers for translation and water transfer of a water heptamer on $\operatorname{Ag}(111)$ on an applied electric field. The approximate conditions of the direct IET experiments displayed in Fig. 1 correspond to $\mathrm{a}+1$ to $+2 \mathrm{~V} / \mathrm{nm}$ field. A positive field pushes electrons into the slab and thus corresponds to a negative tunneling bias voltage. Note that all points correspond to fully optimized reaction barriers at the value of the applied field shown. Initial, transition, and final state structures are also shown. 
puted barriers is not large and could easily be within the error bar associated with the density functional methodology employed. However, more interesting and likely to be more reliable than the result at zero field is the qualitative behavior observed as the electric field is applied. Specifically, we find that the barrier for the water transfer path increases linearly with applied positive field, whereas the barrier for the translation process decreases linearly. The two barriers cross at $\sim+0.5 \mathrm{~V} / \mathrm{nm}$, and, once a field of $\sim+1$ to $+2 \mathrm{~V} / \mathrm{nm}$ is reached, which corresponds to the regime in the direct IET experiments (Fig. 1), there is a reasonably large preference $(0.1-0.2 \mathrm{eV})$ for translation over reorientation. Thus, the DFT calculations are qualitatively consistent with the STM observations, indicating that, in the presence of a positive electric field, translation is favored over internal hydrogen bond rearrangement. Furthermore, examination of the optimized DFT structures along the reaction pathways allows the qualitative trends in the barrier heights with applied fields to be understood. The two diffusion paths considered (Fig. 3) involve transition states with qualitatively different atomic structures, which induce different responses to the external field; in particular, the hopping mechanism involves a transition state with a water molecule relatively high above the surface, whereas the diffusion mechanism does not.

Returning to the experimental results, we address one final interesting feature of the data displayed in Fig. 2. Some, but not all, of the clusters near an excitation site rearrange. Furthermore, it is not even the closest clusters to the point of excitation that are altered. Similar behavior is displayed in Fig. 4, where excitation induces a reorientation of the two clusters marked (1) and (2) but not others such as (3) and (4), which are closer to the point of
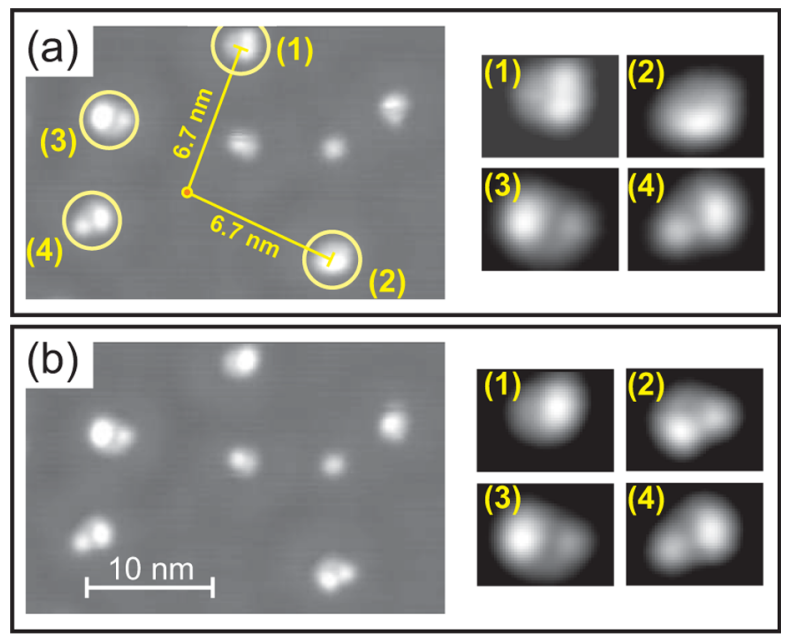

FIG. 4 (color online). Indirect IET manipulation of $\mathrm{D}_{2} \mathrm{O}$ clusters containing 6-12 molecules on $\mathrm{Ag}(111)$. (a) Before manipulation $(V=70 \mathrm{mV}, I=12 \mathrm{pA})$ and (b) after manipulation with $I=12 \mathrm{pA}$ and $V=150 \mathrm{mV}$ for $5.8 \mathrm{~s}$ at the point marked by a small circle in (a). Encircled clusters are shown magnified by 2.5 on the right-hand side. excitation. To further understand and quantify this somewhat surprising observation, the indirect manipulation experiments were repeated many times with a range of tipcluster lateral distances. From these experiments, histograms of the excitation probabilities versus tip-cluster lateral distance were obtained, such as the one shown in Fig. 5. At the electron energy shown $(150 \mathrm{meV})$, the excitation probability exhibits a maximum close to zero due to direct excitation and then three distinct maxima at multiples of $d=3.4 \mathrm{~nm}$ [22]. Excitation at other energies yields qualitatively similar behavior. The histogram in Fig. 5 is important because it shows that clusters can at this energy be manipulated at specific discrete distances of up to approximately $10 \mathrm{~nm}$ and altogether up to distances of $20 \mathrm{~nm}$.

The oscillatory distance dependence in the indirect manipulation experiments suggests that the manipulation involves electrons which are injected into the surface state band of $\operatorname{Ag}(111)$, rather than a direct field effect or the excitation by hot ballistic electrons [10]. Electrons injected into the surface state enter a wave function which is associated with an oscillatory density distribution around the injection point that was previously shown to influence the diffusion behavior of adsorbed atoms [23,24]. The de Broglie wavelength of electrons in the surface state band close to the Fermi energy $E_{F}$ can be calculated via $\left.\lambda=h / \sqrt{2 m^{*}\left(E_{e}-E_{S S}\right.}\right)$, with $h$ the Planck constant and $E_{e}$ the electron energy with respect to the Fermi level. For $\operatorname{Ag}(111)$, the surface state is well characterized with the surface state energy onset at $E_{S S}=-65 \mathrm{meV}$ and the dispersion for energies smaller than $2 \mathrm{eV}$ in good agreement with a free electron with an effective mass of $m^{*}=$ $0.4 m_{e}$ [25]. Thus $\lambda_{F}\left(E_{e}=0\right)=7.4 \mathrm{~nm}$ for $\operatorname{Ag}(111)$. The de Broglie wavelength of the electrons of highest energies used in the experiment reported in Fig. 5 is $\lambda\left(E_{e}=\right.$ $150 \mathrm{meV})=4.2 \mathrm{~nm}$, and thus during the experiment electrons with wavelengths between 7.4 and $4.2 \mathrm{~nm}$ are injected

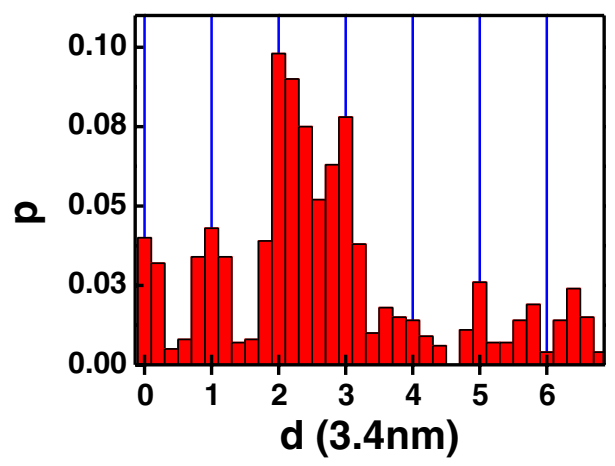

FIG. 5 (color online). Histogram of reaction probability $p$ versus distance $d$ of the cluster from the point of electron injection for manipulation with $150 \mathrm{meV}$. The reaction probability is obtained by dividing the number of clusters observed to change at a particular distance by the total number of clusters at that distance. The $x$ axis scale is in multiples of $3.4 \mathrm{~nm}$. 
into the surface. The oscillation found in the histogram of Fig. 5 corresponds to $\lambda=(6.8 \pm 0.3) \mathrm{nm}$, a value that lies within this wavelength range and corresponds to electrons with an energy of $E_{e}=(17 \pm 7) \mathrm{meV}$. This energy is within the typical range for intermolecular vibrations of metal-supported ice structures [26]. We suggest, therefore, that excitation of intermolecular vibrational modes may initiate the rearrangement of the molecules within the clusters.

In conclusion, we have demonstrated how to selectively induce diffusion of $\mathrm{D}_{2} \mathrm{O}$ nanoclusters across the surface with direct IET manipulation and diffusion of molecules within them with indirect IET, indicating that the variation of the lateral distance of the tip to the manipulated cluster can be used to control the outcome of molecular-scale IET manipulation. With the help of DFT calculations, the difference between conventional IET and indirect IET is rationalized through a strong field-dependent sensitivity to the relative heights of the relevant reaction barriers. A strong distance-dependent sensitivity in the reaction probability for indirect IET manipulation leads to the suggestion that the excitation is mediated by electrons in the surface state band that are resonant with the excited vibration. It seems likely that the indirect IET approach for molecular manipulation at surfaces will not simply be restricted to the water-ice clusters examined here on $\operatorname{Ag}(111)$. Rather, this approach should be feasible whenever there is a surface state band or resonance at the energy that is related to vibrational modes that trigger surface reactions. Hence a multitude of follow-up experiments could be envisioned, e.g., selective switching of molecules at certain distances in molecular arrays even of several molecules at the same time. We further suggest that the manipulation of the surface state, e.g., via quantum corrals [1] or on irregular small terraces [27], can be used to control the distance at which the molecules are manipulated.

H. G. and K. M. acknowledge financial support from the Deutsche Forschungsgemeinschaft. J. C. is grateful to the Alexander von Humboldt Foundation for financial support. A. M.'s work is supported by the EURYI scheme (see [28]) and by the EPSRC.

[1] M. F. Crommie, C. P. Lutz, D. M. Eigler, and E. J. Heller, Surf. Rev. Lett. 2, 127 (1995).

[2] W. Ho, J. Chem. Phys. 117, 11033 (2002).

[3] B. C. Stipe, M. A. Rezaei, and W. Ho, Science 280, 1732 (1998); H. J. Lee and W. Ho, Science 286, 1719 (1999); J. I. Pascual, J. J. Jackiw, Z. Song, P. S. Weiss, H. Conrad, and H.-P. Rust, Phys. Rev. Lett. 86, 1050 (2001); Y. Kim, T. Komeda, and M. Kawai, Phys. Rev. Lett. 89, 126104 (2002).

[4] T. Komeda, Y. Kim, M. Kawai, B. N. J. Persson, and H. Ueba, Science 295, 2055 (2002).
[5] K. Morgenstern and K.-H. Rieder, Chem. Phys. Lett. 358, 250 (2002).

[6] K. Morgenstern and K.-H. Rieder, J. Chem. Phys. 116, 5746 (2002).

[7] J. I. Pascual, N. Lorente, Z. Song, H. Conrad, and H.-P. Rust, Nature (London) 423, 525 (2003).

[8] K. Morgenstern, H. Gawronski, M. Mehlhorn, and K.-H. Rieder, J. Mod. Opt. 51, 2813 (2004).

[9] H. Gawronski, K. Morgenstern, and K.-H. Rieder, Eur. Phys. J. D 35, 349 (2005).

[10] P. Maksymovych, D. B. Dougherty, X.-Y. Zhu, and J. T. Yates, Phys. Rev. Lett. 99, 016101 (2007).

[11] M. Mehlhorn, H. Gawronski, L. Nedelmann, A. Grujic, and K. Morgenstern, Rev. Sci. Instrum. 78, 033905 (2007).

[12] A. Michaelides and K. Morgenstern, Nature Mater. 6, 597 (2007).

[13] L. J. Whitman, J. A. Stroscio, R. A. Dragoset, and R. J. Celota, Science 251, 1206 (1991).

[14] The DFT calculations were carried out within the periodic supercell approach as implemented in the VASP code [15]. The Perdew, Burke, and Ernzerhof [16] exchangecorrelation functional was employed along with the projector augmented wave method [17]. The valence electrons were expanded in a plane wave basis up to a cutoff of $415 \mathrm{eV}$. As in our previous studies [12,18], $\mathrm{Cu}(111)$ was modeled with a three-layer slab in a $p(5 \times 5)$ supercell with a $2 \times 2 \times 1$ Monkhorst-Pack $\boldsymbol{k}$ point mesh. The atoms in the bottom $\mathrm{Cu}$ layer were held fixed, while all other atoms were allowed to relax. Transition state structures have been located with the climbing image nudged elastic band algorithm [19] and electric field effects treated with the scheme introduced by Feibelman [20,21].

[15] G. Kresse and J. Hafner, Phys. Rev. B 47, 558 (1993); G. Kresse and J. Furthmüller, Phys. Rev. B 54, 11169 (1996).

[16] J. P. Perdew, K. Burke, and M. Ernzerhof, Phys. Rev. Lett. 77, 3865 (1996); 78, 1396 (1997).

[17] G. Kresse and D. Joubert, Phys. Rev. B 59, 1758 (1999).

[18] A. Michaelides, Faraday Discuss. 136, 287 (2007).

[19] G. Henkelman, B. P. Uberuaga, and H. Jonsson, J. Chem. Phys. 113, 9901 (2000).

[20] P. J. Feibelman, Phys. Rev. B 64, 125403 (2001).

[21] T. R. Mattsson, B. S. Swartzentruber, R. Stumpf, and P. J. Feibelman, Surf. Sci. 536, 121 (2003).

[22] The intensity variation in the histograms reflects the finite image size. The widths of the maxima originate partly from the clusters' finite width of approximately $1 \mathrm{~nm}$.

[23] J. Repp, F. Moresco, G. Meyer, K.-H. Rieder, P. Hyldgaard, and M. Persson, Phys. Rev. Lett. 85, 2981 (2000).

[24] K. Morgenstern, K.-F. Braun, and K.-H. Rieder, Phys. Rev. Lett. 93, 056102 (2004).

[25] O. Jeandupeux, L. Bürgi, A. Hirstein, H. Brune, and K. Kern, Phys. Rev. B 59, 15926 (1999).

[26] M. A. Henderson, Surf. Sci. Rep. 46, 1 (2002).

[27] K. Morgenstern, K.-H. Rieder, and G. A. Fiete, Phys. Rev. B 71, 155413 (2005).

[28] http://www.esf.org/euryi. 